\title{
Benchmark Dose Calculations for Methylmercury- Associated Delays on Evoked Potential Latencies in Two Cohorts of Children
}

\section{Citation}

Murata, Katsuyuki, Esben Budtz-Jorgensen, and Philippe Grandjean. 2002. “Benchmark Dose Calculations for Methylmercury-Associated Delays on Evoked Potential Latencies in Two Cohorts of Children." Risk Analysis 22 (3) (June): 465-474. doi:10.1111/0272-4332.00034.

\section{Published Version}

doi:10.1111/0272-4332.00034

\section{Permanent link}

http://nrs.harvard.edu/urn-3:HUL.InstRepos:34787283

\section{Terms of Use}

This article was downloaded from Harvard University's DASH repository, and is made available under the terms and conditions applicable to Other Posted Material, as set forth at http:// nrs.harvard.edu/urn-3:HUL.InstRepos:dash.current.terms-of-use\#LAA

\section{Share Your Story}

The Harvard community has made this article openly available.

Please share how this access benefits you. Submit a story.

\section{Accessibility}




\title{
Benchmark Dose Calculations for
}

\section{Methylmercury-Associated Delays}

\author{
on Evoked Potential Latencies
}

\section{in Two Cohorts of Children}

Katsuyuki Murata, ${ }^{1}$ Esben Budtz-Jørgensen, ${ }^{2}$ Philippe Grandjean ${ }^{3,4}$

${ }^{1}$ Department of Hygiene, Akita University School of Medicine, 1-1-1 Hondo, Akita 010-8543, Japan

${ }^{2}$ Department of Biostatistics, Panum Institute, University of Copenhagen, DK-2200 Copenhagen, Denmark

${ }^{3}$ Institute of Public Health, University of Southern Denmark, DK-5000 Odense, Denmark

${ }^{4}$ Departments of Neurology and Environmental Health, Boston University Schools of Medicine and Public Health,

Boston, MA 02118

Editorial correspondence to:

Philippe Grandjean, University of Southern Denmark, Winslowparken 17, 5000 Odense, Denmark

Phone: +45-6550.3769. Fax: +45-6591.1458. Email: pgrandjean@ health.sdu.dk

Short title: Benchmark dose for mercury 
Delays in evoked potential latencies were observed at increased exposures to methylmercury from seafood in two cohorts of children. Because this outcome parameter appeared to be virtually independent of confounders, including cultural differences, a joint analysis of benchmark doses was carried out. Comparable cohort members included 382 Faroese and 113 Madeiran children without middle ear infection or neurological disease at age 7 years. Maternal hair-mercury concentrations at parturition in the Faroese children ranged from 0.6 to $39.1: \mathrm{g} / \mathrm{g}$ (geometric average, $4.49: \mathrm{g} / \mathrm{g}$ ). In Madeira, mothers who had not changed their diet since pregnancy had current hair mercury concentrations ranging from 1.1 to $54.4: \mathrm{g} / \mathrm{g}$ (geometric average $10.14: \mathrm{g} / \mathrm{g}$ ). The mercury-associated delay in peak III latencies at two frequencies $(20$ and $40 \mathrm{~Hz})$ showed similar regression equations in the two groups of children, and benchmark dose calculations were therefore carried out for the two groups separately and jointly. For a doubling of a $5 \%$ prevalence of abnormal results of the peak III latencies at $40 \mathrm{~Hz}$ in a linear dose-response model, the benchmark dose for the maternal hair mercury concentration was $8.79: \mathrm{g} / \mathrm{g}$ for the Faroese children; $8.04: \mathrm{g} / \mathrm{g}$ for the Madeiran children; and $9.46 \mathrm{~g} / \mathrm{g}$ for both groups. Results were similar for the $20 \mathrm{~Hz}$ condition. Benchmark dose results were substantially lower using a logarithmic or square root curve function, although the difference in fit between the curves was far from statistically significant. The benchmark results using evoked potential latencies are in close agreement with results based on neuropsychological test performance.

KEY WORDS: Methylmercury; benchmark dose; brainstem auditory evoked potentials; neurotoxicity; human health risk assessment 


\section{INTRODUCTION}

Methylmercury is a well documented neurotoxicant, which can cause serious adverse effects on brain development, especially when exposures occur prenatally. ${ }^{1,2}$ While current risk assessment efforts have emphasized neuropsychological test results as important outcome variables, ${ }^{2,3}$ delays in evoked potential latencies recorded from the brain have provided useful supplementary evidence of pathological changes in methylmercury poisoning. ${ }^{4-8}$ The neurophysiological response parameters require only minimal cooperation by the subject and are unlikely to be affected by socioeconomic or cultural differences. ${ }^{9}$ Recent studies have reported latency delays at increased exposures to methylmercury from seafood and freshwater fish. ${ }^{10-12}$ This evidence suggests that the latency of peak III on the auditory brainstem response may be particularly sensitive to mercury.

We have been involved in epidemiological studies of 7-year-old children in two geographically separate fishing communities exposed to methylmercury from contaminated seafood. ${ }^{11,12}$ In both island cohorts, increased latencies of evoked potentials were associated with higher maternal exposures but were not affected by the child's current exposure. ${ }^{11,12}$ In the Faroe Islands, increased maternal exposures originate mainly from pilot whale meat, ${ }^{13}$ while the exposure in Madeira is from certain species of marine fish, such as black scabbard. ${ }^{14}$ Both communities are rather stable and homogeneous, and the participation rate in the studies was high. Because the children were examined by similar methods and by the same clinical team, a joint analysis of the data seemed indicated.

Dose-response modeling for risk assessment purposes often uses benchmark dose calculations, ${ }^{15}$ and substantial information is now available for mercury-associated effects on neurological development and performance on psychological tests. ${ }^{2,16-18}$ The current study was therefore carried out to determine benchmark dose levels using similar default conditions for the neurophysiological results and to apply the benchmark approach for joint analysis of results from two separate studies. Because the shape of the dose-response functions for methylmercury neurotoxicity is unknown, ${ }^{2}$ different curve functions were applied.

\section{MATERIALS AND METHODS}

\subsection{Study Populations and Exposure Assessment}

The two studies have been described in detail elsewhere. ${ }^{11,19}$ In the Faroes, the prospective study included 382 children (187 boys and 195 girls) from a birth cohort generated in 1986-1987 and examined at age 7 years in 1993; six children did not complete the examination (one child at $20 \mathrm{~Hz}$ only), and children with neurological disease $(\mathrm{N}=6)$ or current middle ear infection $(\mathrm{N}=45)$ were excluded. ${ }^{12}$ The mercury exposure 
biomarkers available for these children included the mercury concentration in maternal hair obtained at parturition (geometric mean, $4.52: \mathrm{g} / \mathrm{g}$; range, 0.6-39.1:g/g). This parameter is thought to reflect the maternal exposure during pregnancy and is therefore also a risk indicator for fetal neurotoxicity. While the children were also exposed to PCB, this neurotoxicant did not show any clear association with peak latencies. ${ }^{20}$

The cross-sectional study in Madeira involved children in first grade at the two schools at the fishing harbor of Câmara de Lobos, Madeira, Portugal. ${ }^{11}$ The children examined in 1994 were born in 1987. Two children were excluded because of neurological disease; middle ear infection was not present. As indicator of prenatal methylmercury exposure, we used the current mercury concentration in maternal scalp hair. Children whose mothers had changed their diet since pregnancy were therefore excluded $(\mathrm{N}=29)$. For the remaining 113 children (53 boys and 60 girls), the maternal hair mercury concentration varied from 1.1 to 54.4 :g/g (geometric mean, $10.14: \mathrm{g} / \mathrm{g})$.

The mercury analyses of the Faroese samples involved the length of hair strands thought to represent the full pregnancy period, i.e., in most cases $8-9 \mathrm{~cm}$. The analysis was carried out by an experienced laboratory which has also been responsible for similar analyses in other population studies. ${ }^{21}$ For the Madeira children, a 3 -cm proximal hair segment was analyzed by a similar method. ${ }^{11}$ Both laboratories have documented high analytical quality, and the methods are thought to be entirely comparable. The total analytical imprecision has been estimated to be about $3-4 \%$ (as coefficient of variation) at the average hair-mercury concentrations seen in the two populations. $^{11}$

\subsection{Evoked Potential Assessment}

For determination of evoked potential latencies, we used a 4-channel electromyograph (Medelec Saphire-4ME). BAEP was measured in the children in comfortable reclined position. Click signals with an intensity of $65 \mathrm{~dB}$ HL ( $0.1 \mathrm{~ms}$ impulses of alternating polarity) were presented to the right ear through earphones at a rate of first $20 \mathrm{~Hz}$, then $40 \mathrm{~Hz} .{ }^{11,22}$ The other ear was masked with white noise at $45 \mathrm{~dB}$ HL. The BAEP was recorded using three standard EEG electrodes placed on the vertex, the right mastoid ipsilateral to stimulation and the left mastoid (ground). The responses were averaged 1,024 times after amplification and filtration, with one replication at each condition. Peaks I, III and V reflect the volume-conducted electric activity from the acoustic nerve, pons and midbrain, respectively. ${ }^{23}$ Delays in peak I and subsequent peaks may therefore occur in the case of middle ear infection. 
The imprecision of latency assessments was determined from the coefficient of variation of duplicate measures in the Faroese children. The latency of peak I showed imprecisions of $14.6 \%$ and $16.0 \%$ at 20 and $40 \mathrm{~Hz}$, respectively, while the corresponding imprecisions of Peak III latencies were $8.4 \%$ and 7.6\%. Daily examinations over a 16-day period had previously shown coefficients of variation of the peak I latency that were more than twice the level of peak III. ${ }^{24}$ Although the interpeak latency I-III would be preferable to avoid interference from inner ear disease, ${ }^{22}$ we therefore used the total peak III latency as outcome parameter in the present study.

\subsection{Regression Analyses}

Regression analyses were performed using the maternal hair mercury concentration as the dose parameter. The distribution of the mercury results was skewed, and an improved fit of the dose-effect association was obtained using logarithmically transformed mercury data. ${ }^{11,12,19}$ Sex and age were included as potential confounders, although they had only a small effect on the response variables. Other covariates, such as maternal smoking and alcohol intake during pregnancy, were far from significant as predictors of the latencies and were therefore not included in the multiple regression analyses. ${ }^{11,12}$

\subsection{Benchmark Calculations}

As proposed by Crump, ${ }^{15}$ the benchmark dose (BMD) is the dose of a substance that results in an increased probability of abnormal test performance by a benchmark response (BMR), i.e., from $\mathrm{P}_{0}$ for an unexposed child to $\mathrm{P}_{0}+\mathrm{BMR}$ for a child at the BMD. Previous applications of this method ${ }^{2,16-18,25,26}$ have used a $\mathrm{P}_{0}$ of $5 \%$, i.e., an abnormal test performance defined by a probability of $5 \%$ in an unexposed population. This definition of $\mathrm{P}_{0}$ does not take into account any medical considerations. A lower confidence limit (BMDL) for BMD is then calculated as the statistical 95\% lower bound of the BMD. Thus, the BMDL depends on the statistical uncertainty of the BMD determination and will therefore tend to increase when the size of a study increases. Recent calculations have used a BMR of $5 \%$ as an appropriate choice, as it corresponds to a doubling of the prevalence of abnormal test performance. ${ }^{2,26}$ As the magnitude of the BMR substantially affects the calculations, we have also used BMRs of $2 \%$ and $10 \%$.

The benchmark concept was originally developed in the context of highly standardized carcinogenesis trials with a dichotomous outcome. ${ }^{15}$ In applying the method for graded responses in epidemiological studies, the influence by confounders must be taken into account in addition to the exposure of interest. The preferable 
adaptation of the benchmark concept to this situation is to use the same unexposed risk $\left(\mathrm{P}_{0}\right)$ for each child and a linear multivariate model for the dependence on the confounders. Accordingly, for each child the level of abnormal test performance depends on the individual values of the confounders for that child. This approach avoids dependence of the BMD and BMDL on the confounders.

In the two studies considered, the dose is expressed as the mercury concentration in maternal hair. A proper regression model would then be:

$$
\mathrm{Y}_{\mathrm{i}}=\forall+\exists_{1} * \operatorname{sex}_{\mathrm{i}}+\exists_{2} * \operatorname{age}_{\mathrm{i}}+:\left(\mathrm{d}_{\mathrm{i}}\right)+,{ }_{\mathrm{i}},
$$

where $Y_{i}$ denotes the response of child $i$, and $\exists$ is the regression coefficient for a confounder, while the error term ${ }_{\mathrm{i}}$ is normally distributed with a mean of zero. The function : $\left(\mathrm{d}_{\mathrm{i}}\right)$ gives the expected change in latency at the child's dose level $\mathrm{d}_{\mathrm{i}}$ compared to an unexposed child with the same confounder values. Values of BMD and BMDL may be calculated using a statistical dose-response model where : is a power function ${ }^{15}$ for the dependence of a child's expected latencies of evoked potentials on the mercury dose parameter (d):

$$
:(\mathrm{d})=\exists \cong \mathrm{d}^{\mathrm{K}}
$$

The power parameter $\mathrm{K}$ is restricted to values equal to or above 1, thus allowing the dose-response curve to be nonlinear. This approach has the advantage of avoiding unreasonably low BMDLs, which may otherwise occur if the dose-response curve becomes infinitely steep at low doses. However, the parameters do not enter the model in a linear fashion, thus complicating the calculation of the BMDL. We therefore used the parametric bootstrap $\operatorname{method}^{27}$ for this purpose. Two-thousand new data sets were simulated from the distribution of the original data. Each of these data sets has covariate values equal to the original data, whereas the response is given by the function of the covariates plus the random error. Each simulation allows a determination of BMD, and the BMDL is then derived as their 5 th percentile.

Using this approach, a power of 1 generally provided the best fit to the Faroese neuropsychological test data, i.e., a linear association between dose and response. ${ }^{2,26}$ In accordance with the recent applications, ${ }^{2,26}$ we therefore also included the linear dose-response curve. Because of the difference in calculations, the BMDL may be slightly different from the one estimated for the $\mathrm{K}$ power function with $\mathrm{K}=1$.

A logarithmic dose parameter provided a slightly better fit for neuropsychological outcome parameters ${ }^{24}$ and had been used in previous regression analyses of the evoked potential data from both populations. ${ }^{11,12}$ In examining the robustness of the benchmark calculations, we therefore also used a logarithmic and a square root dose parameter. To avoid problems with an infinite slope of these curves at zero dose, the dose 
was transformed after addition of 1 to the mercury concentration. The following three additional dose models were therefore used:

$$
\text { Linear dose-response model: :(d) }=\exists \cong \mathrm{d}
$$

Square root dose-response model: :(d) $=\exists \cong\{\sqrt{(d+1)}-1\}$

Logarithmic dose-response model: :(d) $=\exists \cong \log (\mathrm{d}+1) \quad$ The fits of these three models was determined pairwise, i.e., each model was tested against an expanded model that also included another model. A high p-value in this test indicates that the model at hand does not fit the data significantly worse than the expanded model. Because the expanded model in all cases fitted the data only slightly better than the best of the individual models, the three models can be compared simply by calculating their $-2 \log (\mathrm{L})$ difference and then judging this value from a chi-square table. The fit of the K-power model could not be compared to the rest of the models by formal asymptotic likelihood testing. However, as the K-power model gives approximately the same fit as the linear model but includes one more parameter, at best this model can be considered only as good as the linear model.

\section{RESULTS}

Three hundred and eighty-two Faroese children and 113 Madeiran children without middle ear infection or nervous system disease were included in the benchmark calculations. The mercury concentrations in maternal hair at child-birth were significantly related to the III peak latencies at both 20 and $40 \mathrm{~Hz}$ after adjustment for age and sex (Table I) as previously reported. ${ }^{11,12}$ The average latencies were slightly greater at the Faroes than at Madeira.

When comparing the two regression equations with confounder adjustments, the residual variance was found to be similar. More importantly, the regression coefficients (betas) for mercury did not differ, as suggested by the standard deviations. The lowest p-value for a difference between the two groups of children was 0.08 for the $40 \mathrm{~Hz}$ results using the logarithmic dose scale. Likewise, the small effects of age and sex were similar in all equations compared.

The two sets of data were therefore collapsed, while allowing for different intercepts for the two groups. Table I shows the joint regression coefficients for the logarithmic transformation of the mercury concentration. The effects of mercury measured on the linear or the square root dose scales also showed p-values of 0.001 or below. Figs 1 and 2 depict the association of peak III latencies at the two test conditions with the maternal 
hair-mercury concentration after adjustment of the response variable for age and sex. A logarithmic dose scale has been used because of the skewed distribution of the data.

Tables II-III show the results of the benchmark calculations for peak III latencies of the BAEPs at 20 and $40 \mathrm{~Hz}$ using the four curve models. As expected from Table I, the $20 \mathrm{~Hz}$ results for the Faroes and Madeira are quite similar, but the BMDs and BMDLs are lower in Madeira than in the Faroes for the $40 \mathrm{~Hz}$ test condition. The collapsed population results show BMDs close to the average for the two populations, but the BMDLs are closer to or slightly greater than the largest of the two individual results.

The $\mathrm{K}$ power model showed the best fit at $\mathrm{K}=1$ or slightly above 1 . The BMDs and BMDLs were therefore quite similar to those obtained with the linear dose function. The fit of the logarithmic models linear, square root, and logarithmic models were compared from likelihood function results (data not shown). Despite the fact that the benchmark results in Tables II and III are clearly different, the difference in model fits was far from statistically significant.

\section{DISCUSSION}

The developing brain is thought to constitute the most vulnerable organ in regard to methylmercury exposure. ${ }^{1-3}$ Emphasis in risk assessment has therefore been placed on the neurological function of children with developmental exposure to this neurotoxicant. However, results of psychological tests may not be comparable between cultures and languages, and age standardization may not be meaningful if tables from a different country are employed. In this regard neurophysiological tests may be preferable to the extent that they can be applied in population studies. Assessment of evoked potential latencies is one such method, which has been shown to be sensitive to methylmercury ${ }^{4-8}$ and other neurotoxicants. ${ }^{9,28}$ In the two different populations of 7 -year-old children examined, we found that indicators of prenatal methylmercury exposure were significantly associated with the peak III latency of the BAEPs. The peak III latency reflects axonal conduction in auditory tracts rather than synaptic activation within brainstem auditory nuclei, and delays may imply segmental demyelination and axonal and neuronal loss. ${ }^{23}$ Accordingly, evoked potential latencies are used for clinical purposes in diagnosing early stages of diseases such as multiple sclerosis. ${ }^{23}$ The magnitude of mercury-associated delays is similar to effects previously attributed to exposure to other neurotoxicants. ${ }^{9,28-30}$ For example, delayed BAEP latencies were documented in asymptomatic children exposed to lead, thus suggesting subclinical pathology of the auditory pathway rostral to the cochlear nucleus. ${ }^{29,30}$ Although the latency results obtained have not revealed any frank 
abnormality in the children examined, the evidence on methylmercury neurotoxicity and previous findings on BAEP latencies indicate methylmercury may cause adverse effects on the auditory tracts.

The maternal hair-mercury concentration is probably a rather imprecise measure of the amount of methylmercury that has reached the child's brain during the critical time period of maximum vulnerability. ${ }^{31}$ Although uncertainty about the effective dose in general will affect the validity of the benchmark results, current error models $^{32}$ are not easily applicable for estimation of the possible magnitude of any bias. Some general observations should be noted in this regard. While the Madeira study was cross-sectional in design, information on developmental exposure levels was obtained at birth for the Faroese cohort. The range of mercury exposure in both groups spanned approximately two orders of magnitude, thus possibly limiting the impact of exposure misclassification. Despite the different sampling times for the exposure biomarker, the two populations showed similar and statistically significant mercury-associated delays in peak III latencies. It is noteworthy that mercury exposure levels differed between mother and child, but only the maternal exposure level was a significant risk factor for delayed evoked potential latencies in the child. ${ }^{11,12}$ In other communities where individual differences in exposure levels were thought to be relatively stable, cross-sectional studies of children have also shown clear associations between current mercury exposure levels and neurobehavioral deficits. ${ }^{33,34}$

The cross-cultural similarity of the mercury-association in the two populations is in accordance with the assumption that evoked potential latencies are independent of language, education, and socioeconomic factors. ${ }^{21}$ However, the total peak III latency was about $0.1 \mathrm{~ms}$ shorter in Madeira than in the Faroes, i.e., a difference corresponding to the size of the regression coefficients shown in Table I. Because mercury exposures in Madeira were higher than in the Faroes, this difference cannot be attributed to mercury. Other factors, including genetic differences or temperature, ${ }^{23}$ must be suspected. This finding emphasizes the importance of including subjects with low-level exposures from each population as a built-in control group.

Two different conditions of the BAEP were used. The benchmark results based on the Faroese data are very similar for the two conditions. At $20 \mathrm{~Hz}$, the results obtained for the linear model are slightly higher when based on the Madeira results, but the reverse was seen at the $40 \mathrm{~Hz}$ condition. However, these differences are well within the uncertainties expected from the standard deviations of the regression coefficients (Table I). When merging the data, the resulting BMDs are quite similar to the averages for the two groups if account is taken that the Faroese population was three times larger than the Madeiran. However, the joint BMDL results are closer to the larger of the two, in some cases greater, as the combined number of observations causes a decrease in the 
statistical uncertainty.

In the original approach to benchmark calculations, ${ }^{15}$ a power function was proposed for the dose-response curve, with the power being greater or equal to 1 . When using neuropsychological test results ${ }^{25}$ as the outcome variable, the best fit was obtained at a power of 1, e.g., a linear association. The present study showed similar results, although two of six $\mathrm{K}$ values are slightly above 1 . Accordingly, the benchmark results obtained for the power function are almost the same as those seen with the linear function and does not provide a better fit.

Because the shape of the dose-response curve will depend on the outcome variable and the range of exposures covered, concave curves may also be envisaged. As in previous calculations, ${ }^{25}$ we therefore included for comparison a logarithmic and a square root function. Due to the skewed distribution of mercury exposures and the improved fit obtained, the log transformation had been used in the original analysis of the neurobehavioral data from the two studies. ${ }^{11,12,19,20}$ The BMDL results are much lower for the logarithmic curve shape than for the linear one, with the square root results being intermediate between the two. Although no statistically significant difference in curve fits was observed, this finding emphasizes the substantial dependence of the BMDL results on the default conditions chosen. The same observation was made when considering the neuropsychological outcomes as dependent variables, ${ }^{25}$ and these results therefore emphasize the need to obtain better $a$ priori information on the dose-effect functions.

We also determined visual evoked potentials (VEPs) in both populations. ${ }^{11,12,19}$ However, VEP latencies are known to be affected by nutritional factors, including the supply of essential fatty acids during early development. ${ }^{35}$ Nutritional factors were not assessed in our studies, where delayed VEP peaks were significantly associated with mercury exposure in Madeira, ${ }^{11}$ but not in the Faroes. ${ }^{12}$ In Madeira, the most sensitive VEP parameter seemed to be the N145 peak at the $15^{\prime}$ condition. ${ }^{11}$ Although these results are therefore not included in the present paper, we carried out supplementary benchmark calculations for this parameter in Madeira children who were not wearing glasses. At a BMR of 5\%, the BMDL for the linear model is $12.3: \mathrm{g} / \mathrm{g}$ (data not shown). This result is very similar to the results obtained for the BAEP latencies.

A BMDL of approximately $10: \mathrm{g} / \mathrm{g}$ maternal hair is similar to recently calculated BMDLs for other neurological outcome variables in the Faroese children ${ }^{2,25}$ and in a New Zealand population. ${ }^{2,17}$ Higher BMDLs were reported from a study in the Seychelles, where clear effects on psychological tests have not been detected so far. ${ }^{2,18}$ However, in the absence of a detected mercury effect, the Seychelles results may not be directly comparable with those from studies with clear evidence of mercury toxicity. Using several curve functions, an average BMDL 
of about 10 :g/g was also calculated for crude neurological abnormalities in children exposed in connection with the poisoning incident in Iraq. ${ }^{3,16}$ The most sensitive neurological, neuropsychological, and neurophysiological endpoints therefore seem to agree in regard to the BMDL.

When applying the BMDL results for standard setting, an uncertainty factor must be used., ${ }^{2,3,26}$ The BMDL should not be considered an approximate threshold. Thus, with a BMR of $5 \%$ as the default condition, the BMD corresponds to the dose that causes a doubling of the prevalence of abnormal results. While no medical information has been included in determining the definition of an abnormal result, current evidence on neurotoxicant effects on $\mathrm{BAEP}^{9,28-30}$ would suggest that early adverse effects may be detectable by this outcome parameter. When defining a safe exposure level, an uncertainty factor will therefore be necessary, and the magnitude of this factor must take into account the steepness of the dose-response curve below the BMD. If one chooses the linear dose-response function, the uncertainty factor must also take into account that much lower BMDLs are obtained using other curve functions that may be equally appropriate. Taking into regard as well differences in individual susceptibility and the possibility that other organs could potentially be more vulnerable than the developing brain, authorities have chosen an uncertainty factor of $10 .^{2,3,26}$ Although several studies now offer substantial support for a methylmercury BMDL corresponding to approximately 10 :g/g hair, much less evidence is available to guide the choice of the uncertainty factor.

\section{ACKNOWLEDGMENTS}

Supported by grants from the U.S. National Institute of Environmental Health Sciences (ES06112 and ES09797), the European Commission (Environment Research Programme) and the Danish Medical Research Council. The contents of this paper are solely the responsibility of the authors and do not necessarily represent the official views of the NIEHS, NIH or any other funding agency. 


\section{REFERENCES}

1. International Programme on Chemical Safety (1990). Methylmercury (Environmental Health Criteria 101). Geneva: World Health Organization.

2. National Research Council (2000). Toxicological Effects of Methylmercury. Committee on the Toxicological Effects of Methylmercury, Washington, DC: National Academy Press.

3. U.S.EPA (Environmental Protection Agency) (1997). Mercury Study Report to Congress. Washington, DC.

4. Tokuomi, H., Uchino, M., Imamura, S., Yamanaga, H., Nakanishi, R., \& Ideta, T. (1982). Minamata disease (organic mercury poisoning): neuroradiologic and electrophysiologic studies. Neurology, 32, 1369-1375.

5. Hamada, R., Yoshida, Y., Kuwano, A., Mishima, I., \& Igata, A. (1982). Auditory brainstem responses in fetal organic mercury poisoning (in Japanese). Shinkei-Naika, 16, 282-285.

6. Inayoshi, S., Okajima, T., \& Imai, H. (1988). Electrophysiological studies on Minamata disease (in Japanese). Japanese Journal of EEG \& EMG, 16, 48-56.

7. Inayoshi, S., Okajima, T., Sannomiya, K. \& Tsuda, T. (1993) Brainstem and middle auditory evoked potentials in Minamata disease (in Japanese). Clinical Encephalography, 35, 588-592.

8. Musiek, F.E., \& Hanlon, D.P. (1999). Neuroaudiological effects in a case of fatal dimethylmercury poisoning. Ear and Hearing, 20, 271-275.

9. Araki, S., \& Murata, K. (1993). Determination of evoked potentials in occupational and environmental medicine: a review. Environmental Research, 63, 133-147.

10. Counter, S.A., Buchanan, L.H., Laurell, G., \& Ortega, F. (1998). Blood mercury and auditory neuro-sensory responses in children and adults in the Nambija gold mining area of Ecuador. Neurotoxicology. 19, 185-196.

11. Murata, K., Weihe, P., Renzoni, A., Debes, F., Vasconcelos, R., Zino, F., Araki, S., Jørgensen, P.J., White, R.F., \& Grandjean, P. (1999). Delayed evoked potentials in Madeiran children exposed to methylmercury from seafood. Neurotoxicology and. Teratology, 21, 343-348.

12. Murata, K., Weihe, P., Araki, S., Budtz-Jørgensen, E., \& Grandjean, P. (1999). Evoked potentials in Faroese children prenatally exposed to methylmercury. Neurotoxicology and Teratology, 21, 471-472.

13. Juhlshamn, K., Andersen, A., Ringdal, O., \& Mørkøre, J. (1987). Trace elements in the Faroe Islands I. Element levels in edible parts of pilot whales (Globicephalus meleanus). Science of the Total Environment, $65,53-62$

14. Gaggi, C., Zino, F., Duccini, M., \& Renzoni, A. (1996). Levels of mercury in scalp hair of fishermen and their 
families from Camara de Lobos-Madeira (Portugal): a preliminary study. Bulletin of Environmental Contamination and Toxicology, 56, 860-865.

15. Crump, K. (1995). Calculation of benchmark doses from continuous data. Risk Analysis, 15, 79-89.

16. Crump, K., Viren, J., Silvers, A., Clewell, H., Gearhart, J., \& Shipp, A. (1995) Reanalysis of dose-response data from the Iraqi methylmercury poisoning episode. Risk Analysis, 15, 523-532.

17. Crump, K.S., Kjellström, T., Shipp, A.M., Silvers, A., \& Stewart, A. (1998). Influence of prenatal mercury exposure upon scholastic and psychological test performance: bench-mark analysis of a New Zealand cohort. Risk Analysis, 18, 701-713.

18. Crump, K.S., Van Landingham, C., Shamlaye, C., Cox, C., Davidson, P.W., Myers, G.J., \& Clarkson, T.W. (2000). Benchmark concentrations for methylmercury obtained from the Seychelles Child Development Study. Environmental Health Perspectives, 108, 257-263.

19. Grandjean, P., Weihe, P., White, R.F., Debes, F., Araki, S., Yokoyama, K., Murata, K., Sørensen, N., Dahl, R., \& Jørgensen, P.J. (1997). Cognitive deficit in 7-year-old children with prenatal exposure to methylmercury. Neurotoxicology and Teratology, 19, 417-428.

20. Grandjean, P., Weihe, P., Burse, V.W., Needham, L.L., Storr-Hansen, E., Heinzow, B., Debes, F., Murata, K., Simonsen, H., Ellefsen, P., Budtz-Jørgensen, E., Keiding, N., \& White, R.F. (2001). Neurobehavioral deficits associated with PCB in 7-year-old children prenatally exposed to seafood neurotoxicants. Neurotoxicology and Teratology (in press).

21. Cernichiari, E, Toribara, T.Y., Liang, L., Marsh, D.O., Berlin, M.W., Myers, G.J., Cox, C., Shamlaye, C.F., Choisy, O., \& Davidson, P. (1995). The biological monitoring of mercury in the Seychelles study. Neurotoxicology, 16, 613-628.

22. Chiappa, K.H. (1997). Brainstem auditory evoked potentials: methodology. In K.H. Chiappa, (Ed.), Evoked Potentials in Clinical Medicine, 3rd ed. (pp. 157-197). Philadelphia, PA: Lippincott-Raven.

23. Chiappa, K.H. \& Hill, R.A. (1997) Brainstem auditory evoked potentials: interpretation. In K.H. Chiappa, (Ed.), Evoked Potentials in Clinical Medicine, 3rd ed. (pp. 199-268). Philadelphia, PA: Lippincott-Raven.

24. Murata, K., Araki, S., \& Aono, H. (1990). Central and peripheral nervous system effects of hand-arm vibration tool operations: a study of brain-stem auditory evoked potential and peripheral nerve conduction. International Archives of Occupational and Environmental Health, 62, 183-188.

25. Budtz-Jørgensen, E., Grandjean, P., Keiding, N., White, R.F., \& Weihe, P. (2000). Benchmark Dose 
calculations of methylmercury-associated neurobehavioural deficits. Toxicology Letters, 112-113, 193-199.

26. U.S. Environmental Protection Agency (2001). Water Quality Criterion for the Protection of Human Health: Methylmercury (EPA-823-R-01-001). Washington, DC: U.S.EPA, Office of Science and Technology.

27. Efron, B., \& Tibshirani, R.J. (1993). An Introduction to the Bootstrap. London: Chapman \& Hall.

28. Otto, D.A., \& Hudnell, H.K. (1990). Electrophysiological systems for neurotoxicity testing: PEARL II and alternatives. In B. Johnson, W.K. Anger, A. Durao, \& C. Xintaras (Eds), Advances in Neurobehavioral Toxicology: Applications in Environmental and Occupational Health (pp. 259-276). Chelsea, MI: Lewis.

29. Otto, D., Robinson, G., Baumann, S., Schroeder, S., Mushak, P., Kleinbaum, D., \& Boone, L. (1985). 5-year follow-up study of children with low-to-moderate lead absorption: electrophysiological evaluation. Environmental Research 38, 168-186.

30. Holdstein, Y., Pratt, H., Goldsher, M., Rosen, G., Shenhav, R., Linn, S., Mor, A., \& Barkai, A. (1986). Auditory brainstem evoked potentials in asymptomatic lead-exposed subjects. Journal of Laryngology and Otology 100, 1031-1036.

31. Grandjean P, Jørgensen PJ, Weihe P. Validity of mercury exposure biomarkers. Progress in Nucleic Acid Research and Molecular Biology (in press).

32. Fuller, W.A. (1987). Measurement Error Models. New York: Wiley.

33. Grandjean, P., White, R.F., Nielsen, A., Cleary, D., \& de Oliveira Santos, E.C. (1999). Mercury neurotoxicity in Amazonian children downstream from gold mining. Environmental Health Perspectives, 107, 587-591.

34. Cordier, S., Garel, M., Amiel-Tison, C., Morcel, H., \& Mandereau, L. (1999). Neurologic and neurodevelopment investigations of methylmercury-exposed children in French Guinea. Epidemiology, 10, S102.

35. Jensen, C.L., Prager, T.C., Fraley, J.K., Chen, H., Anderson, R.E., \& Heird, W.C. (1997). Effect of dietary linoleic/alpha-linolenic acid ratio on growth and visual function of term infants. Journal of Pediatrics, 131, 200-209. 
Figure legends:

Fig. 1. Peak III latency on the brainstem auditory evoked potentials at $20 \mathrm{~Hz}$ for 382 Faroese and 113 Madeiran children aged 7 years in relation to the maternal hair-mercury concentration. The slopes of the two regression lines are not statistically different.

Fig. 2. Same as Fig. 1 but at the $40 \mathrm{~Hz}$ condition. 
Table I. Average (and Standard Deviation, SD) Brainstem Auditory Evoked Potential Peak III Latencies (in ms) in 382 Faroese and 113 Madeiran Children Without Middle Ear Infection or Neurological Disease after Adjustment for Age and Sex, and the Regression Coefficients (Betas with SD) for the Logarithmic Transformation of the Mercury Concentration in Maternal Hair

$\begin{array}{llccc}\text { Frequency } & \text { Population } & \text { Mean (SD) } & \text { Beta (SD) } & \text { P Value } \\ & & & & \\ & \text { Faroes } & & & \\ & \text { Madeira } & 4.18(0.27) & 0.113(0.042) & 0.008 \\ & \text { Both } & 4.09(0.29) & 0.165(0.079) & 0.038 \\ 40 \mathrm{~Hz} & \text { Faroes } & 4.16(0.28) & 0.122(0.037) & 0.001 \\ & \text { Madeira } & 4.32(0.31) & 0.111(0.048) & 0.022 \\ & \text { Both } & 4.23(0.35) & 0.294(0.094) & 0.002\end{array}$


Table II. Benchmark Dose (BMD in :g Mercury/g of Hair) and its Lower 95\% Confidence Limit (BMDL) at Different Benchmark Response (BMR) Levels and According to Four Different Dose-response Models for BAEP Peak III Latency at $20 \mathrm{~Hz}$ in 382 Faroese, 113 Madeiran Children, and Both Groups

\begin{tabular}{|c|c|c|c|c|c|c|c|}
\hline \multirow[t]{2}{*}{ Model } & & Power $^{1)}$ & \multicolumn{2}{|c|}{ Linear } & Square root & \multicolumn{2}{|c|}{ Logarithmic } \\
\hline & & BMD BMDL & BMD $\mathrm{F}$ & BMDL & BMD BMDL & BMD & BMDL \\
\hline $\mathrm{BMR}=0.02$ & Faroes & 10.704 .62 & 5.55 & 3.47 & 2.841 .54 & 1.11 & 0.58 \\
\hline & Madeira & $9.13 \quad 5.25$ & 9.13 & 4.89 & $3.40 \quad 1.54$ & 0.83 & 0.39 \\
\hline & Both & $7.26 \quad 5.04$ & 7.26 & 4.80 & 3.141 .84 & 1.05 & 0.61 \\
\hline $\mathrm{BMR}=0.05$ & Faroes & $\begin{array}{ll}16.31 & 9.59\end{array}$ & 11.94 & 7.45 & $8.37 \quad 4.17$ & 3.98 & 1.68 \\
\hline & Madeira & 19.6211 .18 & 19.62 & 10.52 & $10.28 \quad 4.19$ & 2.67 & 1.03 \\
\hline & Both & 15.6010 .72 & 15.60 & 10.32 & 9.385 .13 & 3.69 & 1.78 \\
\hline $\mathrm{BMR}=0.10$ & Faroes & 21.6814 .83 & 20.00 & 12.48 & 18.838 .82 & 13.72 & 4.20 \\
\hline & Madeira & 32.8518 .53 & 32.85 & 17.61 & 23.508 .85 & 7.81 & 2.27 \\
\hline & Both & 26.1217 .86 & 26.12 & 17.29 & 21.2811 .05 & 12.30 & 4.53 \\
\hline
\end{tabular}

\footnotetext{
${ }^{1)} \mathrm{K}=1.81$ for Faroes, and 1 for Madeira and both
} 
Table III. Benchmark Dose (BMD in :g Mercury/g of Hair) and its Lower 95\% Confidence Limit (BMDL) at Different Benchmark Response (BMR) Levels and According to Three Different Dose-response Models for BAEP Peak III Latency at $40 \mathrm{~Hz}$ in 381 Faroese, 113 Madeiran Children, and Both Groups

Model

$\begin{array}{cccc}\text { Power }^{1)} & \text { Linear } & \text { Square root } & \text { Logarithmic } \\ \text { BMD BMDL } & \text { BMD BMDL } & \text { BMD BMDL } & \text { BMD BMDL }\end{array}$

$\begin{array}{ccccccccc}\text { BMR=0.02 Faroes } & 7.33 & 4.43 & 7.33 & 4.09 & 3.91 & 1.83 & 1.43 & 0.66 \\ \text { Madeira } & 5.87 & 4.01 & 5.87 & 3.74 & 1.91 & 1.11 & 0.49 & 0.29 \\ \text { Both } & 6.79 & 4.66 & 6.38 & 4.40 & 2.76 & 1.70 & 0.96 & 0.57 \\ \text { BMR=0.05 Faroes } & 15.75 & 9.41 & 15.75 & 8.79 & 12.04 & 5.08 & 5.76 & 1.98 \\ \text { Madeira } & 12.61 & 8.60 & 12.61 & 8.04 & 5.34 & 2.90 & 1.34 & 0.73 \\ \text { Both } & 14.20 & 9.97 & 13.71 & 9.46 & 8.11 & 4.68 & 3.23 & 1.65 \\ \text { BMR=0.10 Faroes } & 26.37 & 15.42 & 26.37 & 14.72 & 27.85 & 10.93 & 23.55 & 5.22 \\ \text { Madeira } & 21.11 & 14.37 & 21.11 & 13.47 & 11.55 & 5.94 & 3.16 & 1.50 \\ \text { Both } & 23.33 & 16.59 & 22.96 & 15.83 & 18.19 & 9.99 & 10.21 & 4.12\end{array}$

\footnotetext{
${ }^{1)} \mathrm{K}=1$ for Faroes and Madeira, and 1.04 for both
} 